\title{
DESIGUALDAD DE OPORTUNIDADES EN MÉXICO: IMPACTO SOBRE EL CONSUMO Y SOBRE LAS HABILIDADES COGNITIVAS
}

\author{
Diana Alférez Esperón* \\ David Mayer-Foulkes* \\ (Recibido: Febrero 2011 / Aprobado: Mayo 2011)
}

\begin{abstract}
RESUMEN
Utilizando información de la encuesta ENNVIH 2002 y 2005, se estima una cota inferior de la desigualdad de oportunidades para el gasto en el consumo de los hogares y para el nivel de habilidades cognitivas de la población en México. El estudio atribuye una parte de la desigualdad a características que están fuera del control de los individuos. Circunstancias como lugar de nacimiento, género, pertenencia a un grupo indígena y escolaridad de los padres se utilizan para calcular índices de desigualdad. Los resultados muestran que la desigualdad de oportunidades es de aproximadamente $35 \%$ en el gasto en el consumo y de $50 \%$ en el nivel de habilidades cognitivas.

Palabras clave: Desigualdad de oportunidades, consumo, capacidad cognitiva, justicia distributiva, México

Clasificación JEL: D31, D63
\end{abstract}

\footnotetext{
* Centro de Investigación y Docencia Económicas. Correos electrónicos:<diana.ae@gmail.com>, <david.mayer@cide.edu>.
} 


\begin{abstract}
Using information from ENNVIH 2002 and 2005, we estimate a lower bound for inequality of opportunities for Mexican household consumption expenditure and cognitive ability. The study attributes a portion of inequality to characteristics beyond the individual's control. Circumstances such as place of birth, gender, belonging to an indigenous group and father's schooling are used to estimate inequality indices. The results show that inequality of opportunities is approximately $35 \%$ in consumption expenditure and 50\% in cognitive ability.

Keywords: Inequality opportunities, consumption, cognitive ability, distributive justice, Mexico

JEL Classification: JEL: D31, D63
\end{abstract}

\title{
1. INTRODUCCIÓN
}

El desarrollo humano es uno de los principales aspectos del desarrollo a nivel global. La publicación del Human Development Report (HDR) de 1990 tuvo como principal objetivo destacar el papel central del ser humano como protagonista y objetivo del proceso de desarrollo, en términos del debate económico y político. De acuerdo al Programa de las Naciones Unidas para el Desarrollo, el desarrollo humano es un paradigma que va mucho más allá del aumento o la disminución de los ingresos de un país. Comprende la creación de un entorno en el que las personas puedan desarrollar su máximo potencial y llevar adelante una vida productiva y creativa de acuerdo con sus necesidades e intereses. El desarrollo implica ampliar las oportunidades para que cada persona pueda vivir una vida que valore. ${ }^{1}$ De esta forma, el desarrollo humano se define como un proceso de largo plazo donde la dinámica intergeneracional juega un papel esencial en la transmisión y

\footnotetext{
${ }^{1}$ Concepto de desarrollo humano que proporciona el Programa de las Naciones Unidas para el Desarrollo. Revisado el 30 de enero de 2011, <http:/hdr.undp.org/es/desarrollohumano/>.
} 
AlfÉrez EsPerón / MAYER-FoulKes Desigualdad de oportunidades en México:

Impacto sobre el consumo y sobre las habilidades cognitivas

formación de las capacidades humanas. Uno de los problemas que enfrenta este proceso intergeneracional es el de la desigualdad de oportunidades.

La desigualdad de oportunidades económicas crea diferencias en las condiciones iniciales del desarrollo humano. La teoría de igualdad de oportunidades considera esencial nivelar estas diferencias, para que las circunstancias como el lugar de nacimiento, el género, la etnia a la que se pertenece o los antecedentes familiares - circunstancias que quedan más allá del control y responsabilidad de los individuos- no determinen su horizonte de posibilidades de vida.

En general, existen dos posturas diferentes acerca de la igualdad de oportunidades en la literatura. La primera se relaciona con la meritocracia (Lucas, 1995; Arrow et al. 2000). Este enfoque postula que los individuos son completamente responsables de la situación en la que se encuentran -su ingreso, salud o situación de empleo depende completamente de ellos-. La segunda postura considera que hay una parte de la vida del individuo que es función de variables que quedan más allá de su control -por ejemplo sus antecedentes socioeconómicos-y otra parte que está determinada por su esfuerzo -por ejemplo las horas que decide trabajar- ${ }^{2}$ El presente trabajo toma como base la segunda postura, que mira como iguales a todas las personas. Existen diversos estudios sobre los determinantes de la desigualdad de oportunidades en el ingreso o consumo del hogar y en los grados de estudio alcanzados por los individuos. Sin embargo, poco se ha estudiado en economía sobre la desigualdad de oportunidades en las habilidades cognitivas.

El objetivo de este trabajo es medir la magnitud de la desigualdad de oportunidades en las habilidades cognitivas y el gasto en el consumo en México. Para ello se tomará como referencia el artículo de Ferreira y Gignoux (2008), The Measurement of Inequality of Opportunity: Theory and an application to Latin America, y se utilizarán datos a nivel individuo de la Encuesta Nacional de Niveles de Vida de los Hogares (ENNVIH). Con esta información se construirá una medida de la desigualdad en el consumo y otra en el nivel de habilidades cognitivas; es decir, se estimará la proporción de la desigualdad que puede ser atribuida a

\footnotetext{
${ }^{2}$ Véase como ejemplo Roemer (1993, 1998, 2006), Van de Gaer (1993), Fleurbaey (1995), Roemer et al. (2003), Lefranc et al. (2006), Ferreira et al. (2008) y Barros et al. (2009).
} 
las circunstancias que quedan fuera del control del individuo y la proporción que puede ser atribuida a su esfuerzo.

Este tipo de trabajo es relevante ya que permite analizar la capacidad de la sociedad de aumentar las oportunidades de los grupos con más desventajas y ofrecer un nivel de participación más igualitario. Dado que la reducción de la desigualdad económica constituye uno de los principales retos para el desarrollo humano y para el desarrollo económico, es fundamental comprender la desigualdad de oportunidades en México para poder analizar las posturas acerca de la redistribución, las cuales influencian directamente el marco de las políticas públicas.

El trabajo se divide de la siguiente manera: en la primera parte, se realiza una descripción del concepto de desigualdad de oportunidades y una breve revisión bibliográfica sobre diversos estudios que la analizan. En la segunda parte se describe el marco teórico y el modelo. A continuación se muestran las estadísticas descriptivas relevantes y por último, se presentan resultados y conclusiones.

\section{EL CONCEPTO DE DESIGUALDAD DE OPORTUNIDADES}

De acuerdo al ideal de justicia social e igualdad, todos los seres humanos deben tener los mismos derechos y ser tratados como iguales. En la literatura, existen distintas teorías sobre la justicia distributiva y diferentes contextos en los que se analiza la igualdad de ingreso, bienestar, habilidades, libertad, oportunidades o salario. Dworkin (1981) plantea que la justicia implica compensar a los individuos por los aspectos de la situación en la que se encuentran, por los cuales no son responsables y obstaculizan su desempeño. La compensación sólo debe darse en estos aspectos ya que las diferencias entre los factores por los que son responsables no le conciernen a la justicia.

Entre los bienes que son deseables de igualar en una sociedad, existen algunos que impiden llegar a un punto de perfecta equidad. Dado que hay distintas estrategias de igualación y distintos bienes por los cuales una sociedad puede llegar a ser más equitativa, es importante distinguir aquellos bienes que pueden asignarse, aquellos que no pueden asignarse pero su distribución puede modificarse a través 
Alférez EsPerón / MAYer-Foulkes Desigualdad de oportunidades en México:

Impacto sobre el consumo y sobre las habilidades cognitivas

de la asignación de otros bienes y aquellos cuya distribución no puede ser afectada. Una vez establecidos estos criterios, una sociedad que se proponga alcanzar el ideal igualitario debe encontrar bienes y estrategias que faciliten el objetivo de equidad. Una de las teorías que se enfoca en analizar la desigualdad es la teoría de igualdad de oportunidades. En ella se estipula que la recompensa de cada individuo debe ser producto de su esfuerzo y no de circunstancias que están fuera de su control. Un ejemplo de estas circunstancias son las condiciones iniciales de las que parten los individuos. De este modo, la teoría de igualdad de oportunidades plantea que una sociedad debe llevar a cabo una política que compense a las personas y las sitúe en la posición que deberían tener de no haber sido por las circunstancias iniciales en las que nacieron.

Existen varios estudios que buscan derivar medidas de desigualdad para la distribución de oportunidades. El marco teórico propuesto por Roemer (1993, 1998) ha sido la base de muchas de estas investigaciones. En su libro Igualdad de Oportunidades, Roemer menciona que es justo que los individuos sean responsables de sus acciones por su grado de esfuerzo, mas no por aquellos aspectos de la efectividad del esfuerzo que resulten de sus circunstancias. En su artículo Equilizing Opportunity Through Educational Finance Reform, este autor plantea una metodología que debe llevarse a cabo antes de elaborar una política económica enfocada a la desigualdad de oportunidades:

(1) Identificar las circunstancias, aspectos del entorno del individuo que están fuera de su control y afectan su bienestar.

(2) Separar a la población en $k$ tipos dependiendo de las circunstancias que existan, de tal manera que los individuos de un mismo tipo tengan las mismas circunstancias.

(3) Definir una medida de esfuerzo.

(4) Establecer el factor que se desea igualar.

(5) Elegir el instrumento de política para alcanzar el objetivo.

De acuerdo a Roemer (1998), la política de igualdad de oportunidades debería hacer que el grado por el que un individuo alcanza su objetivo sea independiente 
de sus circunstancias y sea función sólo del esfuerzo que realice. En general, el instrumento puede ser una cantidad de recursos que se asigna a los diferentes grupos. Dado que una cantidad limitada puede no ser suficiente para alcanzar el objetivo de igualar las oportunidades, Roemer (1998) propone una política que maximice la ventaja - la variable en la que se da la desigualdad- del grupo con más desventajas al mismo nivel de esfuerzo relativo.

En una línea de investigación más reciente se encuentran los trabajos de Van de Gaer, Schokkaert y Martinez (2001), Bourguignon, Ferreira y Menéndez (2007), Checchi y Peragine (2005), Lefranc, Pistolesi y Trannoy (2006), Ferreira y Gignoux (2008), y Barros et al. (2009). Las medidas de desigualdad de oportunidades que proponen estos autores consisten en relacionar la distribución de resultados observados (como el ingreso, consumo o grados de educación) a factores observados que no dependen del individuo; como la etnia a la que pertenecen, la ocupación y educación de los padres y el lugar donde nacieron. Esta relación ayuda a medir la contribución de los dos conjuntos de factores que determinan la desigualdad de oportunidades: el que es responsabilidad del individuo (decisiones tomadas, esfuerzo y talento) y el que no lo es (circunstancias iniciales).

Estas medidas se han aplicado a información que proviene de encuestas de varias partes del mundo para analizar la magnitud de la desigualdad de oportunidades. Para la región de América Latina, Bourguignon, Ferreira y Menéndez (2007) y Cogneau y Gignoux (2007) calcularon medidas de desigualdad de oportunidades para la distribución de ingresos en Brasil, mientras que Ferreira y Gignoux (2008) calcularon las mismas medidas para la distribución per cápita del ingreso y consumo del hogar en Colombia, Ecuador, Guatemala, Panamá y Perú. Estos últimos autores hacen hincapié en las diferencias que representa medir la desigualdad de oportunidades mediante el ingreso y mediante el consumo; mencionan que los ingresos y los salarios no se miden tan precisamente como los gastos de consumo (particularmente en las encuestas estándares de ingreso de productores agrícolas auto-empleados o trabajadores del sector informal). Así, el consumo tiene más probabilidades de estar cerca del ingreso permanente que del ingreso corriente (siempre que los hogares tengan acceso a algunos mecanismos que les permitan suavizar el consumo). Estos dos trabajos encuentran 
AlfÉrez EsPerón / MAYER-FoulKes Desigualdad de oportunidades en México: Impacto sobre el consumo y sobre las habilidades cognitivas

que una proporción significativa de la desigualdad económica -entre una quinta parte y una tercera parte de los ingresos y aproximadamente la mitad del consumo- puede ser atribuida a la desigualdad de oportunidades.

Otros estudios muestran la tendencia de la distribución de oportunidades a lo largo del tiempo. En un reporte de la ONU sobre desarrollo humano, Gignoux (2009) trabaja con un conjunto de datos de cinco países (Brasil, Colombia, Ecuador, Panamá y Perú) y estudia la contribución a la distribución de oportunidades en el ingreso de la expansión en la oferta educativa que tuvo lugar entre 1960-1980. Su análisis muestra que la desigualdad en ingreso se redujo en los cinco países durante ese periodo, sin embargo sólo encontró reducciones significativas en la desigualdad de oportunidades en Brasil y Perú.

Para el caso de México, Mayer y Cordourier (2006) plantean un esquema que permite definir políticas de compensación con los criterios de la teoría de igualdad de oportunidades. Con información de la Encuesta Nacional de Ingreso y Gasto de los Hogares (ENIGH) de 1996, desarrollan una metodología que permite medir la desigualdad de oportunidades tomando como caso de estudio la brecha salarial entre mujeres y hombres. Los autores aplican una regresión por cuartiles para los diferentes niveles de ingreso de su muestra y hacen una descomposición de la brecha salarial. Con ello demuestran que una política homogénea de compensación no es la opción más eficiente para alcanzar la igualdad de oportunidades: en el decil más bajo, los hombres son objeto de discriminación, lo cual se debe sobre todo a las deficiencias en su nivel de educación. En los deciles 3 a 9 las mujeres experimentan una discriminación que implica recibir entre $15 \%$ y $18 \%$ menos del salario que recibirían hombres igualmente cualificados. Dado que las mujeres que trabajan cuentan con mayor escolaridad que los hombres, Mayer y Cordourier argumentan que una política de compensación basada en acervos educativos no sería lo más apropiado. En su lugar, políticas que disminuyan la discriminación e igualen las oportunidades de la mujer deberían reducir los prejuicios culturales e institucionales, así como el efecto económico de los papeles biológicos y sociales de la mujer.

Un estudio más reciente sobre México se incluye en el libro Measuring Inequality of Opportunities in Latin America and the Caribbean publicado 
por el Banco Mundial. Uno de los capítulos muestra los resultados de una evaluación que compara la desigualdad económica de oportunidades en Brasil, Colombia, Ecuador, Guatemala, México, Panamá y Perú. Se encontró que la desigualdad de oportunidades económicas representa entre 20\% y $30 \%$ de la desigualdad general de los ingresos laborales. La mayor desigualdad de oportunidades se encontró en Brasil, y la menor en Colombia. Los autores reportan que la clasificación de la desigualdad de oportunidades resultó muy diferente a la clasificación de la desigualdad general de los ingresos laborales. Los resultados indican que, cuando se mide el bienestar de los hogares por el ingreso per cápita del hogar, estimaciones conservadoras de la desigualdad van de $21 \%$ en México a $37 \%$ en Guatemala. Cuando se utiliza el consumo per cápita del hogar, la proporción de la desigualdad total es aún más alta que cuando se considera el ingreso del hogar: 27\% en Colombia y México, 34\% en Ecuador, $35 \%$ en Perú, $42 \%$ en Panamá, y 52\% en Guatemala.

El análisis de los impactos de las políticas públicas en los cambios de la desigualdad de oportunidades es otra rama de estudio sobre este tema. De acuerdo a Gignoux (2009), se está empezando a considerar la contribución de los aumentos en la oferta educativa y las políticas que conciernen a la educación para disminuir la desigualdad de oportunidades. Simulaciones de descomposición sugieren que en Brasil, Colombia y Ecuador, la expansión educativa mejoró notablemente la distribución de oportunidades en el ingreso. Para el caso de México, Behrman, Parker y Todd (2007) analizan los efectos de las transferencias monetarias condicionales del programa Oportunidades. Este programa liga las transferencias monetarias a la inversión en capital humano de las familias con el objetivo de reducir la pobreza y la transmisión intergeneracional de ella. La evaluación del programa después de 5 años mostró que hay impactos positivos en el aumento de grados de escolaridad concluidos por los niños que son parte del programa. En cuanto al trabajo, sus estimaciones muestran una reducción en la tendencia a participar en el mercado laboral a una edad temprana y también muestran un cambio en la estructura de empleos: los jóvenes se están saliendo de empleos agrícolas para ingresar a empleos no-agrícolas. De esta manera, los programas sociales de 
Alférez EsPerón / MAYer-Foulkes Desigualdad de oportunidades en México: Impacto sobre el consumo y sobre las habilidades cognitivas

transferencias monetarias, como este programa gubernamental de asistencia social, podrían ayudar a disminuir la desigualdad causada por las diferencias en oportunidades.

\section{MODELO}

Esta sección describe el marco conceptual que desarrolla el modelo de Roemer (1998) y que utiliza el artículo de Ferreira y Gignoux (2008). Este modelo es el que se utilizará para medir la desigualdad de oportunidades en México. La descripción de los datos que se utilizan se presenta en el siguiente apartado.

Para medir la desigualdad de oportunidades de un determinado resultado, primero se tiene que descomponer la desigualdad total en dos partes: una generada por las circunstancias que están fuera del control del individuo y, otra generada por las diferencias en esfuerzo. Para identificar qué proporción de la desigualdad se debe a las circunstancias y qué proporción se debe a otros factores, primero se deben identificar las variables relacionadas con las circunstancias. Estas variables deben ser exógenas al individuo; es decir, las variables pueden determinar el ingreso del individuo o de su hogar pero su ingreso no puede determinar dichas variables. Por ejemplo, se pueden considerar el género, la raza o etnicidad, el lugar de nacimiento, el nivel educativo alcanzado por la madre, el nivel educativo alcanzado por el padre, y la ocupación principal del padre. Una vez identificadas las variables exógenas, la muestra se divide en grupos de modo que todas las personas que están en un determinado grupo tengan exactamente la misma combinación de circunstancias. Los subgrupos resultantes son conocidos en la documentación científica como tipos. Estos grupos luego son comparados unos con otros. Las diferencias en el resultado -ingreso del hogar o del individuo-entre un grupo u otro pueden ser atribuidas a la desigualdad de oportunidades, mientras que las diferencias de los individuos que se encuentran en un mismo grupo pueden ser consideradas como el resultado del esfuerzo o la suerte.

De manera formal, se considera una población finita de agentes indexados

por $i \in\{1, \ldots, N\}$. Cada individuo $i$ se caracteriza por tener un conjunto de atri- 
butos $\left\{y_{i}, C_{i}, e_{i}\right\}$, donde $y$ denota una ventaja, $C=\left(C_{i}\right)$ es una matriz de características circunstanciales, y $e$ denota el nivel de esfuerzo del individuo. La variable esfuerzo se considera continua, mientras que cada vector $C_{i}$ que representa la circunstancia del individuo $i$, es discreto. Cada entrada $C_{i}^{j}$ representa el aspecto $j$ de las circunstancias de $i$ y toma alguno de un número finito de valores $x_{j}$.

Además, Roemer (1998) asume que $y$ es una función monótona no decreciente del esfuerzo $e$. Este esfuerzo no se observa y su distribución se asume independiente de las circunstancias. Solamente se evalúa la desigualdad por diferencias entre las circunstancias iniciales. Así, Roemer define la igualdad de oportunidades como la situación en que los niveles de ventaja son independientes de las circunstancias. Esta condición ha sido clasificada por Bourguignon, Ferreira y Walton (2007), Ferreira y Gignoux (2008) y Lefranc, Pistolesi y Trannoy (2008) como la definición "estricta" de igualdad de oportunidades.

Foster y Shneyerov (2000), seguidos por Ferreira y Gignoux (2008) y Ferreira, Gignoux y Aran (2010), definen un criterio menos estricto que solamente considera la media de la distribución de ventajas. Al adoptar este criterio se mide la desigualdad de oportunidades de acuerdo a la desigualdad de medias $\mu^{k}(y)$ de ventajas $y$ en diferentes circunstancias $k$. Así, una medida escalar de la desigualdad de oportunidades es una proporción de la desigualdad total en la ventaja, la cual está dada por la división de circunstancias:

$$
\theta_{r}=\frac{I\left(\left\{\mu_{i}^{k}\right\}\right)}{I(y)}
$$

Aquí $I(\cdot)$ es una medida escalar de desigualdad. La ecuación muestra la desigualdad entre grupos como proporción de la desigualdad total en $y$, en donde los grupos son una división de la población total tal que los miembros de cada grupo posean circunstancias idénticas.

Para encontrar una medida $I(\cdot)$ intuitivamente satisfactoria, Ferreira y Gignoux (2008) le requieren tres propiedades básicas: 
Alférez EsPerón / MAYer-Foulkes Desigualdad de oportunidades en México:

Impacto sobre el consumo y sobre las habilidades cognitivas

(1)El índice de desigualdad $I(\cdot)$ satisface el axioma de descomposición path-independence. Es decir que, tomar la desigualdad primero al interior y después entre grupos da el mismo resultado que en el orden inverso. De aquí se deduce

$$
\theta_{r}=\frac{E_{0}\left(\left\{\mu_{i}^{k}\right\}\right)}{E_{0}(y)}
$$

donde $E_{0}$ es la desviación media logarítmica (mean logarithmic deviation).

(2) $\theta_{r}$ satisface los axiomas de réplica de población, invariancia en la escala, normalización y simetría para cada tipo.

(3) No todas las circunstancias relevantes a la ventaja y se observan en los datos. Cualquier división de la población resulta una división incompleta en términos del conjunto total de circunstancias, algunas dentro del alcance de las decisiones del individuo y otras no, que debería incluir el análisis de acuerdo a la teoría. Si se define mejor la división de la población, observando más circunstancias, entonces la proporción de desigualdad queda mejor definida y aumenta. Por lo tanto, $\theta_{r}$ es una cota inferior de la verdadera proporción de desigualdad entre-tipos.

\section{DATOS}

Los datos se obtuvieron de la Encuesta Nacional sobre Niveles de Vida de los Hogares (ENNVIH) 2002 y 2005. Esta encuesta tiene la ventaja de ser representativa a nivel nacional y dar un seguimiento a los hogares que ya fueron encuestados, de tal manera que se pueden llevar a cabo análisis de corte longitudinal. Los cuestionarios de la encuesta están diseñados para capturar variables socioeconómicas tanto a nivel individual como a nivel hogar. De esta manera, se tienen registros del consumo, fuentes de ingresos y activos del hogar, composición de la familia, así como información detallada sobre la escolaridad de un miembro del hogar, historia crediticia y de empleo, registros sobre migración, gustos y hábitos. De acuerdo a Rubalcava y Teruel (2006) la primera ronda de la encuesta, llevada a cabo en 2002, cubrió un total de 8550 hogares y 35 mil 
individuos. En la segunda ronda de la encuesta se re-entrevistaron a los hogares e individuos de la primera ronda; debido a la migración se obtuvo una tasa de re-contacto cercana al $90 \%$ de la muestra original.

De la encuesta se extrajo información sobre: antecedentes familiares (nivel de escolaridad de los padres y ocupación del padre), lugar de nacimiento e información referente a la pertenencia a algún grupo indígena. De acuerdo a Barros et al. (2009), el número de categorías para cada circunstancia no debe ser mayor a tres para que los subgrupos no tengan pocas o nulas observaciones. Este paso es importante ya que el análisis se basa en la calidad de los estimadores para los promedios condicionales de cada grupo. Si los grupos en los que se divide la población contienen pocas observaciones, la variación muestral puede ser muy alta. Esto ocasionaría que se incrementara la desigualdad entre grupos estimada, llevando a una sobreestimación de la desigualdad de oportunidades.

A continuación se hace una breve descripción de las variables que se utilizan:

- Lugar de nacimiento: se divide en dos categorías, haber nacido en una ciudad o haber nacido en un pueblo, ranchería, ejido, hacienda, villa u otro.

- Grupos indígenas: la información se captura mediante la habilidad de hablar una lengua indígena.

- Ocupación del padre: se divide en tres categorías, trabajador en el sector de agricultura, trabajador fuera del sector de agricultura y una última categoría en el que el encuestado reportaba no saber la ocupación de su padre. Esta última categoría representa una distancia con el padre y como tal puede aportar información al análisis sobre la desigualdad económica de oportunidades.

- Escolaridad de los padres: se divide en tres categorías, sin educación, primaria, y secundaria o más.

La Tabla 1 resume las variables que se consideraron con sus respectivos niveles. El número máximo de grupos que puede obtenerse dadas las categorías consideradas y sus respectivos niveles es de 108. La muestra se limitó a individuos que tuvieran 25 años o más al momento de realizar la encuesta. Para la ronda de 2002 se obtuvieron 71 y para la de 2005 se obtuvieron 75 grupos. El promedio de ob- 
Alférez EsPerón / MAYer-Foulkes

Desigualdad de oportunidades en México:

Impacto sobre el consumo y sobre las habilidades cognitivas

\section{TABLA 1}

\section{Definición de ariables circunstanciales}

\begin{tabular}{||l|l|c|}
\multicolumn{1}{c}{ Variables } & Niveles & Categorías \\
\hline Lugar de nacimiento & 2 categorías & Ciudad; áreas rurales \\
Pertenencia a una etnia & 2 categorías & Pertenece a una etnia; no pertenece \\
Educación de la madre & 3 categorías & Sin educación; primaria completa; secundaria o más \\
Educación del padre & 3 categorías & Sin educación; primaria completa; secundaria o más \\
Ocupación del padre & 3 categorías & $\begin{array}{r}\text { Obrero o empleado en el sector agropecuario; otros } \\
\text { sectores; no sabe o no respondió }\end{array}$ \\
\hline
\end{tabular}

servaciones por grupo es de 19.4 y 19.8 para 2002 y 2005, respectivamente. Para las variables de ventajas (variables dependientes) se tomó el gasto mensual en el consumo a nivel del hogar (primera estimación) y el nivel de habilidades cognitivas (segunda estimación). El nivel de habilidades cognitivas se reporta como el porcentaje de respuestas correctas obtenidas por el individuo en la prueba de matrices Raven. ${ }^{3}$ En la Tabla 7 del anexo se encuentra la estadística descriptiva de las variables que se consideraron.

\section{RESULTADOS}

\section{A. DESIGUALDAD DE OPORTUNIDADES EN EL GASTO DEL CONSUMO DEL HOGAR}

El ingreso total del hogar o los gastos en el consumo son algunas medidas de bienestar que se pueden considerar para medir la desigualdad de oportunidades

\footnotetext{
${ }^{3}$ Esta prueba sirve para medir la capacidad de razonamiento y entendimiento de cada individuo de la muestra a través de la asociación de patrones en figuras y colores. Una de las ventajas de esta prueba es que no requiere que el encuestado haya recibido algún tipo de educación. No es necesario que el individuo sepa leer o escribir.
} 
en México. Sin embargo, como se mencionó anteriormente, el consumo tiene más probabilidades de estar cerca del ingreso permanente que del ingreso corriente (siempre que los hogares tengan acceso a algunos mecanismos que les permitan suavizar el consumo). En esta sección se reportan los resultados que se obtienen al considerar el gasto del hogar como la variable de ventaja en términos del modelo de Romer.

El concepto de igualdad de oportunidades del gasto en el consumo requiere que la distribución del gasto, condicional a una variable circunstancial, sea idéntica a la distribución marginal. Es decir, no deberían de existir diferencias entre las distribuciones estimadas para los distintos subgrupos. La Tabla 2 muestra la media de las distribuciones del gasto en el consumo del hogar condicionales a las variables circunstanciales seleccionadas para cada ronda de la ENNVIH.

La diferencia entre las medias muestra una desigualdad entre los niveles de las características seleccionadas. En el caso del lugar de nacimiento, la media del gasto en el consumo del hogar, para ambas rondas de la encuesta, es mayor para las personas que nacieron en una ciudad en comparación de las que nacieron en un pueblo, ranchería, ejido, hacienda o villa. Lo mismo sucede con la distribución del consumo condicional a pertenecer a un grupo indígena. La media es más alta para las personas que no son indígenas. Para los casos del nivel de escolaridad de los padres, la media de la distribución es mayor para aquellos que tuvieron padres que estudiaron secundaria o un nivel mayor, y la media del gasto en el consumo es superior para aquellos con un padre que no trabajaba en el sector agricultura. Dado que las distribuciones entre hombres y mujeres es muy similar, esta variable se excluyó del análisis.

Las medidas de desigualdad que desarrollan Ferreira y Gignoux (2008), y que se presentan en el apartado del modelo teórico, sirven para cuantificar las diferencias que se observan en la Tabla 2. En la siguiente tabla se presentan los resultados que miden la desigualdad de oportunidades en el gasto del consumo del hogar para las dos rondas de la ENNVIH. Cada ronda contiene los estimadores de la desigualdad total en el consumo usando tres índices de entropía generalizada: la desviación media logarítmica, GE(0); el índice de Theil, GE(1); y, un medio de la raíz cuadrada del coeficiente de variación, GE(2). Los índices co- 
AlfÉrez EsPerón / MAYER-FoulKes

Desigualdad de oportunidades en México:

Impacto sobre el consumo y sobre las habilidades cognitivas

\section{TABLA 2}

\section{Media de las distribuciones del consumo del hogar condicionales a las variables circunstanciales seleccionadas}

\begin{tabular}{|c|c|c|c|}
\hline \multirow{2}{*}{\multicolumn{2}{|c|}{ Ronda ENNVIH }} & \multicolumn{2}{|c|}{$\begin{array}{l}\text { Media del gasto mensual en } \\
\text { el consumo del hogar (LN) }\end{array}$} \\
\hline & & 2002 & 2005 \\
\hline \multirow{2}{*}{ Indígena } & Sí & $\begin{array}{c}7.545 \\
{[0.0204]}\end{array}$ & $\begin{array}{c}7.547 \\
{[0.0232]}\end{array}$ \\
\hline & No & $\begin{array}{c}7.935 \\
{[0.0079]}\end{array}$ & $\begin{array}{c}7.978 \\
{[0.0082]}\end{array}$ \\
\hline \multirow{2}{*}{$\begin{array}{l}\text { Lugar de } \\
\text { nacimiento }\end{array}$} & Ciudad & $\begin{array}{c}8.256 \\
{[0.0127]} \\
\end{array}$ & $\begin{array}{c}8.313 \\
{[0.0153]}\end{array}$ \\
\hline & $\begin{array}{l}\text { Pueblo, ranchería, } \\
\text { ejido, hacienda o villa }\end{array}$ & $\begin{array}{l}7.739 \\
{[0.0087]}\end{array}$ & $\begin{array}{l}7.752 \\
{[0.0101]}\end{array}$ \\
\hline \multirow{3}{*}{$\begin{array}{l}\text { Ocupación } \\
\text { del padre }\end{array}$} & Agricultura & $\begin{array}{c}7.611 \\
{[0.0130]}\end{array}$ & $\begin{array}{c}7.646 \\
{[0.0135]}\end{array}$ \\
\hline & No-agricultura & $\begin{array}{c}8.14 \\
{[0.0140]}\end{array}$ & $\begin{array}{c}8.17 \\
{[0.0155]}\end{array}$ \\
\hline & No sabe & $\begin{array}{c}7.937 \\
{[0.0112]}\end{array}$ & $\begin{array}{c}8.008 \\
{[0.0116]}\end{array}$ \\
\hline \multirow{3}{*}{$\begin{array}{l}\text { Educación } \\
\text { del padre }\end{array}$} & Sin educación & $\begin{array}{c}7.727 \\
{[0.0093]}\end{array}$ & $\begin{array}{c}7.772 \\
{[0.0098]}\end{array}$ \\
\hline & Primaria & $\begin{array}{c}8.057 \\
{[0.0122]}\end{array}$ & $\begin{array}{c}8.121 \\
{[0.013]}\end{array}$ \\
\hline & Más & $\begin{array}{c}8.589 \\
{[0.0293]}\end{array}$ & $\begin{array}{c}8.59 \\
{[0.0302]}\end{array}$ \\
\hline \multirow{3}{*}{$\begin{array}{c}\text { Educación } \\
\text { de la } \\
\text { madre }\end{array}$} & Sin educación & $\begin{array}{c}7.718 \\
{[0.0091]}\end{array}$ & $\begin{array}{c}7.77 \\
{[0.0094]}\end{array}$ \\
\hline & Primaria & $\begin{array}{c}8.128 \\
{[0.0122]}\end{array}$ & $\begin{array}{c}8.178 \\
{[0.0136]}\end{array}$ \\
\hline & Más & $\begin{array}{c}8.639 \\
{[0.0332]}\end{array}$ & $\begin{array}{c}8.632 \\
{[0.0360]}\end{array}$ \\
\hline
\end{tabular}

Nota: Errores estándar en paréntesis.

Todas las diferencias entre las distribuciones son significativas al 1\% de significancia de acuerdo a las pruebas Kolmogorov-Smirnov realizadas.

rrespondientes a la desigualdad de oportunidades y a las circunstancias son las medidas relativas a la desigualdad total. La Tabla 3 muestra que, en 2002 la desviación media logarítmica es de 0.394 para la desigualdad total del consumo de los hogares, mientras que el índice de desigualdad de oportunidades es de 0.14. Esto quiere decir que la desigualdad ocasionada por las circunstancias que están 
fuera del control del individuo es aproximadamente $37.79 \%$ de la desigualdad total. En 2005, la desviación media logarítmica es de 0.411 para la desigualdad total del consumo de los hogares, mientras que el índice de desigualdad de oportunidades es de 0.15 . Esto quiere decir que la desigualdad ocasionada por las circunstancias que están fuera del control del individuo es aproximadamente $36.46 \%$ de la desigualdad total de este año. Así, se puede ver que la desigualdad total respecto al gasto en el consumo del hogar aumentó de 2002 a 2005. Sin embargo, la proporción de la desigualdad de oportunidades disminuyó en una pequeña proporción.

\section{TABLA 3}

Índices de desigualdad de oportunidades por gasto en el consumo del hogar

\begin{tabular}{|l|ccc|c|c|c|}
\hline Ronda de la ENNVIH & \multicolumn{3}{|c|}{2002} & & \multicolumn{2}{c|}{2005} \\
\hline Índices & $\mathrm{GE}(0)$ & $\mathrm{GE}(1)$ & $\mathrm{GE}(2)$ & $\mathrm{GE}(0)$ & $\mathrm{GE}(1)$ & $\mathrm{GE}(2)$ \\
\hline Desigualdad total & 0.394 & 0.3995 & 0.6183 & 0.4116 & 0.3951 & 0.5789 \\
& {$[0.0058]$} & {$[0.0069]$} & {$[0.0168]$} & {$[0.0059]$} & {$[0.0066]$} & {$[0.0159]$} \\
Desigualdad de & 0.1489 & 0.1577 & 0.1173 & 0.1501 & 0.1661 & 0.1294 \\
oportunidades & {$[0.0081]$} & {$[0.0093]$} & {$[0.0089]$} & {$[0.0074]$} & {$[0.0091]$} & {$[0.0098]$} \\
Lugar nacimiento & 0.0585 & 0.0609 & 0.0419 & 0.0672 & 0.0744 & 0.0543 \\
& {$[0.0037]$} & {$[0.004]$} & {$[0.0029]$} & {$[0.0048]$} & {$[0.0054]$} & {$[0.004]$} \\
Indígena & 0.0204 & 0.0181 & 0.0106 & 0.0187 & 0.0176 & 0.0109 \\
& {$[0.0028]$} & {$[0.0023]$} & {$[0.0012]$} & {$[0.0027]$} & {$[0.0024]$} & {$[0.0014]$} \\
Educación de la & 0.0762 & 0.0837 & 0.0623 & 0.0653 & 0.075 & 0.0579 \\
madre & {$[0.0056]$} & {$[0.0065]$} & {$[0.0055]$} & {$[0.0049]$} & {$[0.006]$} & {$[0.0051]$} \\
& 0.0754 & 0.0844 & 0.0642 & 0.0613 & 0.0711 & 0.0556 \\
Educación del padre & {$[0.0067]$} & {$[0.0081]$} & {$[0.007]$} & {$[0.005]$} & {$[0.0061]$} & {$[0.0052]$} \\
& 0.0462 & 0.0438 & 0.0275 & 0.0425 & 0.0425 & 0.028 \\
Ocupación del padre & {$[0.0046]$} & {$[0.0044]$} & {$[0.0029]$} & {$[0.0041]$} & {$[0.0041]$} & {$[0.0027]$} \\
\hline
\end{tabular}

Nota: Errores estándar en paréntesis. 
AlfÉrez EsPerón / MAYER-FoUlKes Desigualdad de oportunidades en México: Impacto sobre el consumo y sobre las habilidades cognitivas

La Figura 1 muestra gráficamente la descomposición de los tres estimadores para la desigualdad total en el consumo de los hogares, atribuida a la desigualdad de oportunidades, y un término residual asociado al esfuerzo y suerte de los individuos. En ambas rondas el estimador GE(2) muestra una mayor desigualdad total y una menor desigualdad de oportunidades en términos relativos. Sin embargo, esto se debe a que este estimador es más sensible a las diferencias de la distribución del gasto en el consumo en la parte superior. En general, si el parámetro $\alpha$ del índice es mayor, $\mathrm{GE}(\alpha)$ es más sensible a las diferencias en el consumo en la parte superior de la distribución. Entre menor es $\alpha, \operatorname{GE}(\alpha)$ es más sensible a las diferencias en la

\section{Figura 1}

Descomposición de los tres estimadores para la desigualdad total en el consumo de los hogares

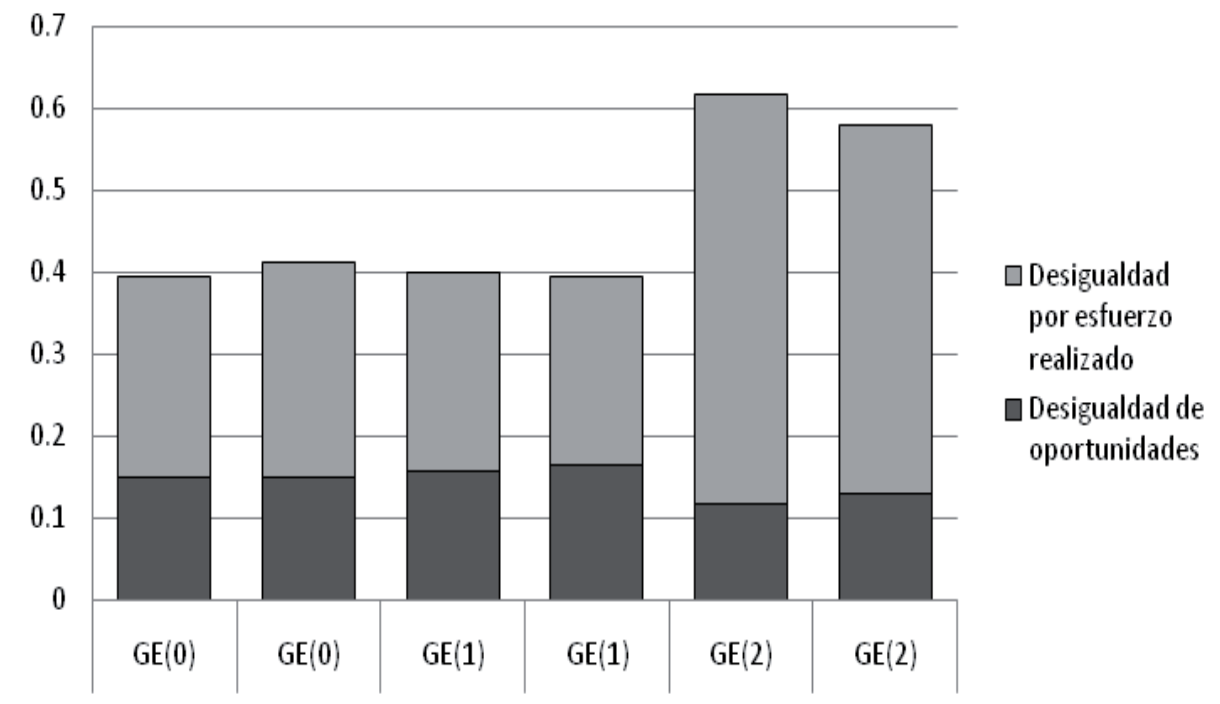


parte inferior de la distribución. Por esta razón GE(2) se puede considerar menos relevante. En la Figura 2 se puede observar que los estimadores GE(0) y GE(1) son similares. En el caso de GE(0), se puede ver gráficamente que la desigualdad total aumentó ligeramente de 2002 a 2005.

Tomando en cuenta el estimador que satisface el axioma de descomposición path-independance, $\mathrm{GE}(0)$, se estimaron, por separado, las proporciones de desigualdad para cada variable del conjunto de circunstancias considerado en este trabajo: lugar de nacimiento, pertenecer a un grupo indígena, nivel de escolaridad de los padres y ocupación del padre.

\section{FigURA 2}

Desigualdad de oportunidades por circunstancias

Comparación entre rondas 2002 y 2005

Estimador GE(0)

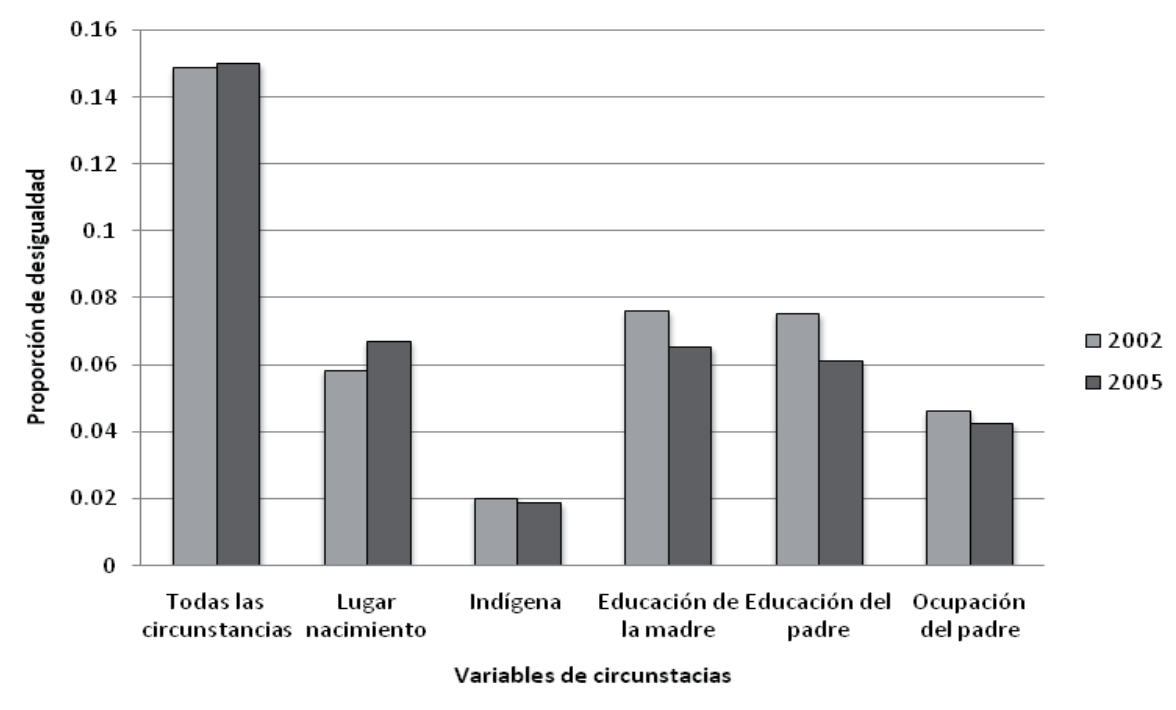


Alférez EsPerón / MAYer-Foulkes Desigualdad de oportunidades en México:

Impacto sobre el consumo y sobre las habilidades cognitivas

Al desagregar las circunstancias se puede observar que, las variables que influyen la desigualdad de oportunidades variaron poco entre 2002 y 2005. En la primera ronda de la ENNVIH, el principal componente está dado por los grados de escolaridad de los padres. Este componente, junto con la ocupación del padre, disminuyó en la encuesta de 2005. Por otro lado, la desigualdad causada por la pertenencia a un grupo indígena es la variable con menor proporción en ambas muestras. El lugar de nacimiento se sitúa como una de las principales causas de la desigualdad de oportunidades en México, incrementando su participación en menos de tres años.

\section{B. DESIGUALDAD DE OPORTUNIDADES EN LAS HABILIDADES COGNITIVAS}

La Encuesta Nacional de Niveles de Vida de los Hogares contiene una sección que reporta la habilidad cognitiva de los individuos a través de una prueba de matrices Raven. Las preguntas del cuestionario pueden interpretarse conforme a una escala del 0 al 100, en el que los individuos que no tuvieron aciertos se codifiquen con 0 , mientras que los individuos con todos los aciertos se marquen con 100. Las habilidades cognitivas, medidas a través de esta prueba, han generado gran discusión acerca de lo que representan. Por un lado, estas habilidades pueden ser una capacidad innata y predeterminada de la capacidad de razonar de los individuos. Por otro lado hay trabajos que argumentan que es posible que las habilidades cognitivas capturen el capital humano adquirido a través del tiempo.

De acuerdo a Mayer et al. (2008) la habilidad cognitiva se puede considerar como un indicador de salud que juega un papel central en la adquisición de la educación. Individuos con un entorno favorable durante su infancia -una mejor alimentación o mejores cuidados- pueden tener un mejor desarrollo en su capacidad de razonamiento. Rubalcava y Teruel (2004) estudian los retornos diferenciables en la salud de los niños de tres fuentes de capital humano materno: escolaridad, habilidades cognitivas y su entorno infantil. Estos autores encuentran que, un entorno más rico y con más infraestructura durante la infancia se encuentra relacionado de manera positiva a una mayor habilidad cognitiva cuando son adultos. 
Dado que las habilidades cognitivas en la edad adulta dependen del entorno en el que se desarrollan los individuos en su infancia, en esta sección se explora la desigualdad de oportunidades debido a las circunstancias en las que nacieron. En este caso, la variable dependiente de interés es el resultado que los encuestados hayan obtenido en la prueba de matrices Raven. Las variables independientes son de nuevo el lugar de nacimiento, la pertenencia a un grupo indígena, el nivel de escolaridad de los padres y la ocupación del padre. En la Tabla 4 se muestran las diferencias entre las medias de las distribuciones para los distintos subgrupos de acuerdo a la variable que representa las habilidades cognitivas.

$\mathrm{Al}$ igual que en la estimación anterior, donde se tomó el gasto en el consumo mensual del hogar, existen diferencias entre los niveles de las características seleccionadas de los individuos. En el caso del lugar de nacimiento, la media de las habilidades cognitivas para ambas rondas de la encuesta es mayor para las personas que nacieron en una ciudad en comparación con las que nacieron en una zona rural. Por otra parte, la media de la distribución es más alta para las personas que no son indígenas. Para los casos del nivel de escolaridad de los padres, la media de la distribución es mayor para aquellos que tuvieron padres que estudiaron secundaria o un nivel mayor y la media de las habilidades cognitivas es superior para aquellos con un padre que no trabajaba en el sector agricultura. La Tabla 5 muestra los resultados de los índices de desigualdad de oportunidades para este caso.

Para cada ronda se reportan los estimadores de la desigualdad total en el nivel de habilidades cognitivas de los tres índices de entropía generalizada y los estimadores de la desigualdad de oportunidades. La Tabla 5 muestra que, en 2002 la desviación media logarítmica es de 0.167 para la desigualdad total en el nivel de habilidades cognitivas, mientras que el índice de desigualdad de oportunidades es de 0.098. Esto quiere decir que la desigualdad ocasionada por las circunstancias que están fuera del control del individuo es aproximadamente 59.11\% de la desigualdad total. En 2005, la desviación media logarítmica es de 0.127 para la desigualdad total, mientras que el índice de desigualdad de oportunidades es de 0.053 . Esto quiere decir que la desigualdad ocasionada por las circunstancias que están fuera del control del individuo es aproximadamente $42.25 \%$ de la desigualdad total. Así, se puede ver que tanto la desigualdad total respecto al nivel 
Alférez EsPerón / MAYer-Foulkes Desigualdad de oportunidades en México: Impacto sobre el consumo y sobre las habilidades cognitivas

de habilidades cognitivas como la proporción de desigualdad de oportunidades disminuyeron de 2002 a 2005.

TABLA 4

Media de las distribuciones de las habilidades cognitivas condicionales a las variables circunstanciales seleccionadas

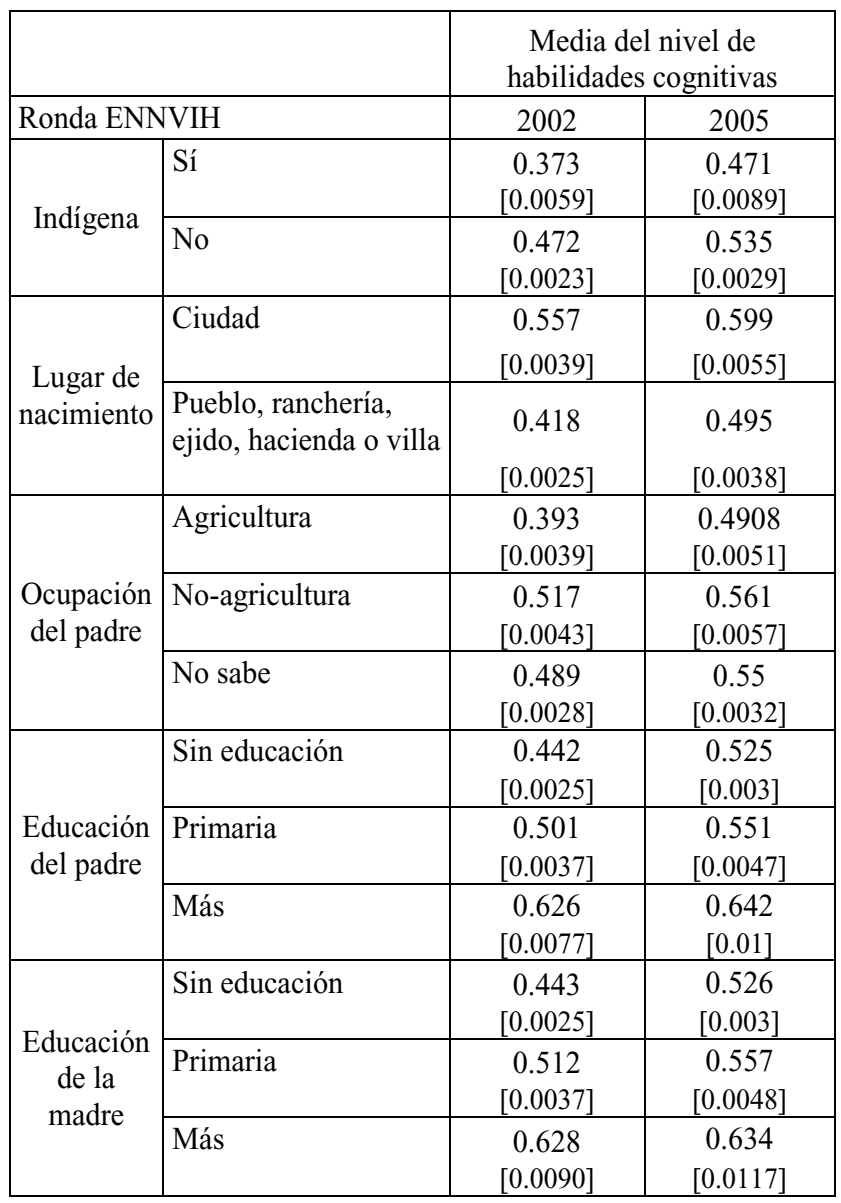


La Figura 3 muestra gráficamente la descomposición de los tres estimadores para la desigualdad total en el nivel de habilidades cognitivas. En este caso el mayor nivel de desigualdad total lo muestra el estimador GE(0). Los estimadores GE(1) y GE(2) son menores que la desviación media logarítmica en los dos años que se presentan. Esto se debe a la sensibilidad del parámetro en las diferencias en la distribución del nivel de habilidades cognitivas. Se puede observar que entre mayor es $\alpha$ de $\mathrm{GE}(\alpha)$, el índice de desigualdad total es menor y el índice de desigualdad de oportunidades es mayor. También se puede apreciar que los tres índices disminuyeron de la primera ronda de la ENNVIH a la segunda, por lo que se ve una reducción de la desigualdad de oportunidades de 2002 a 2005 en los estimadores.

TABLA 5

Índices de desigualdad de oportunidades por nivel de habilidades cognitivas

\begin{tabular}{|c|c|c|c|c|c|c|}
\hline $\begin{array}{l}\text { Ronda de la } \\
\text { ENNVIH }\end{array}$ & \multicolumn{3}{|c|}{2002} & \multicolumn{3}{|c|}{2005} \\
\hline Índices & GE(0) & GE(1) & GE(2) & GE(0) & GE(1) & GE(2) \\
\hline Desigualdad total & $\begin{array}{c}0.1673 \\
{[0.0017]}\end{array}$ & $\begin{array}{c}0.137 \\
{[0.0013]}\end{array}$ & $\begin{array}{c}0.1283 \\
{[0.0014]}\end{array}$ & $\begin{array}{c}0.1271 \\
{[0.0016]}\end{array}$ & $\begin{array}{c}0.1026 \\
{[0.0011]}\end{array}$ & $\begin{array}{c}0.0934 \\
{[0.001]}\end{array}$ \\
\hline $\begin{array}{l}\text { Desigualdad de } \\
\text { oportunidades }\end{array}$ & $\begin{array}{c}0.0989 \\
{[0.0054]}\end{array}$ & $\begin{array}{c}0.1196 \\
{[0.0063]}\end{array}$ & $\begin{array}{c}0.1283 \\
{[0.0068]}\end{array}$ & $\begin{array}{c}0.0537 \\
{[0.0047]}\end{array}$ & $\begin{array}{c}0.0671 \\
{[0.0057]}\end{array}$ & $\begin{array}{c}0.0748 \\
{[0.0064]}\end{array}$ \\
\hline Lugar nacimiento & $\begin{array}{c}0.0479 \\
{[0.0031]}\end{array}$ & $\begin{array}{c}0.0598 \\
{[0.0039]}\end{array}$ & $\begin{array}{c}0.0655 \\
{[0.0043]}\end{array}$ & $\begin{array}{c}0.0275 \\
{[0.0033]}\end{array}$ & $\begin{array}{c}0.0347 \\
{[0.0042]}\end{array}$ & $\begin{array}{c}0.0387 \\
{[0.0047]}\end{array}$ \\
\hline Indígena & $\begin{array}{c}0.0141 \\
{[0.0019]}\end{array}$ & $\begin{array}{c}0.0161 \\
{[0.0021]}\end{array}$ & $\begin{array}{c}0.0162 \\
{[0.002]}\end{array}$ & $\begin{array}{c}0.0047 \\
{[0.0018]}\end{array}$ & $\begin{array}{c}0.0057 \\
{[0.0021]}\end{array}$ & $\begin{array}{c}0.006 \\
{[0.0021]}\end{array}$ \\
\hline $\begin{array}{l}\text { Educación de la } \\
\text { madre }\end{array}$ & $\begin{array}{c}0.0226 \\
{[0.0018]}\end{array}$ & $\begin{array}{c}0.0289 \\
{[0.0024]}\end{array}$ & $\begin{array}{c}0.0324 \\
{[0.0028]}\end{array}$ & $\begin{array}{c}0.0072 \\
{[0.0012]}\end{array}$ & $\begin{array}{c}0.0092 \\
{[0.0016]}\end{array}$ & $\begin{array}{c}0.0105 \\
{[0.0019]}\end{array}$ \\
\hline $\begin{array}{l}\text { Educación del } \\
\text { padre }\end{array}$ & $\begin{array}{c}0.0242 \\
{[0.0021]}\end{array}$ & $\begin{array}{c}0.0312 \\
{[0.0027]}\end{array}$ & $\begin{array}{c}0.0354 \\
{[0.0031]}\end{array}$ & $\begin{array}{c}0.0088 \\
{[0.0016]}\end{array}$ & $\begin{array}{c}0.0115 \\
{[0.0021]}\end{array}$ & $\begin{array}{c}0.0132 \\
{[0.0024]}\end{array}$ \\
\hline $\begin{array}{l}\text { Ocupación del } \\
\text { padre }\end{array}$ & $\begin{array}{c}0.0271 \\
{[0.0022]}\end{array}$ & $\begin{array}{c}0.0319 \\
{[0.0025]}\end{array}$ & $\begin{array}{c}0.033 \\
{[0.0026]}\end{array}$ & $\begin{array}{c}0.0098 \\
{[0.0018]}\end{array}$ & $\begin{array}{c}0.0119 \\
{[0.0022]}\end{array}$ & $\begin{array}{c}0.0128 \\
{[0.0023]}\end{array}$ \\
\hline
\end{tabular}


AlfÉrez EsPerón / MAYER-FoulKes

Desigualdad de oportunidades en México:

Impacto sobre el consumo y sobre las habilidades cognitivas

\section{Figura 3}

\section{Descomposición de los tres estimadores para la desigualdad total en los niveles de habilidades cognitivas}

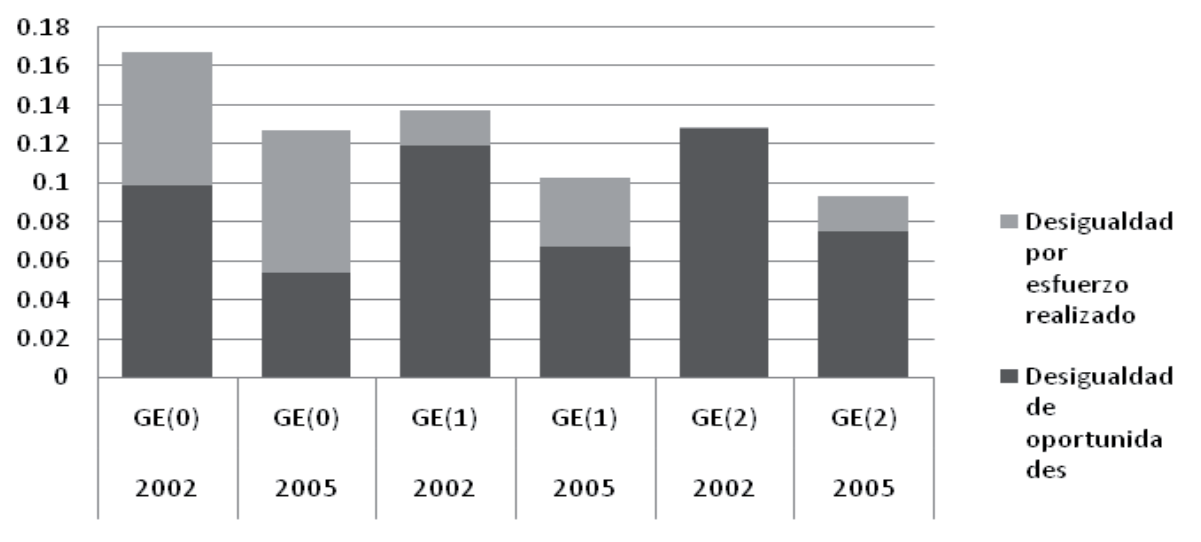

Se pueden observar grandes diferencias al examinar los resultados obtenidos en los índices para las variables de gasto en el consumo del hogar y el nivel de habilidades cognitivas. En comparación con el índice reportado para la desigualdad total en el gasto en el consumo (0.392), la desigualdad en habilidades cognitivas es menor (0.173). Esto puede atribuirse a que los resultados estandarizados de las pruebas de matrices de Raven tienen un valor mínimo de cero y máximo de 1 , mientras que el gasto mensual en el consumo es una variable continua que no está acotada. Sin embargo, en la primera ronda de la ENNVIH el porcentaje de la desigualdad de oportunidades es mucho más alta en el caso de las habilidades cognitivas (55.26\%) que en el caso del gasto en el consumo (35.92\%). Los datos de la segunda ronda muestran una menor diferencia, aún así la desigualdad de oportunidades sigue siendo mayor para el nivel de habilidades cognitivas $(37.05 \%)$ que para el gasto en el consumo (34.76\%). 
En la Figura 4 se pueden observar las estimaciones correspondientes de la desviación media logarítmica, $\mathrm{GE}(0)$, para cada variable considerada en el conjunto de circunstancias.

\section{Figura 4}

\section{Desigualdad de oportunidades por circunstancias \\ Comparación entre rondas 2002 y 2005 \\ Estimador GE(0)}

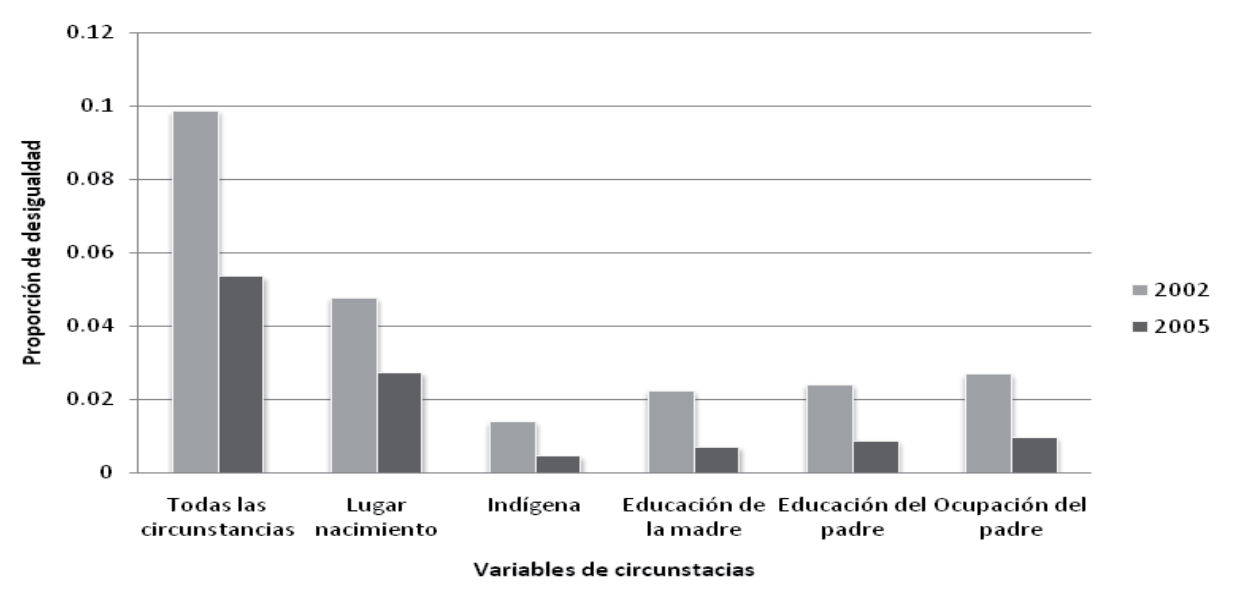

Al desagregar las variables de circunstancia se encuentra que, en 2002 el lugar de nacimiento es la variable que determina gran parte de la desigualdad de oportunidades de los individuos. La educación del padre y su ocupación son los segundos componentes más importantes. En este caso, el nivel de escolaridad de la madre no juega un papel tan importante como lo hacía en el índice de desigualdad de gasto en el consumo. En cuanto al indicador de pertenencia a un grupo indígena, se puede observar que es el indicador que tiene menor participación 
Alférez EsPerón / MAYer-Foulkes Desigualdad de oportunidades en México: Impacto sobre el consumo y sobre las habilidades cognitivas

en la desigualdad en ambas rondas y en ambas estimaciones. Con los datos de la segunda ronda se encuentra el mismo patrón que con los datos de la primera, sin embargo, la proporción de desigualdad es mucho menor en 2005 que en $2002 .{ }^{4}$

Las divisiones de la población en grupos, que se utilizaron para las descomposiciones reportadas en el análisis, se usaron para identificar individualmente a los grupos con más desventajas. Todos los grupos se ordenaron de menor a mayor de acuerdo a la media del consumo o a la media del nivel de habilidades cognitivas del grupo al que pertenecen. Los grupos con más desventajas se identifican como los $m$ grupos que se encuentran en la parte inferior de la distribución del consumo o del nivel de habilidades cognitivas, donde la proporción de la población en $m$ está dada por aquellos que se encuentran en el primer decil. La Tabla 6 muestra los grupos con combinaciones de circunstancias que están en las peores condiciones, de acuerdo a datos de la ENNVIH.

De la población de los $m$ grupos en el primer decil, en ambas rondas se puede observar que alrededor del $35 \%$ es indígena. Sin embargo, los grupos con más desventajas económicas son aquellos que están compuestos por individuos que nacieron en una zona no urbana $(\approx 99 \%)$, cuyos padres no reportan ningún nivel de escolaridad $(\approx 95 \%)$ y cuyo padre trabajaba en una actividad relacionada a la agricultura $(\approx 80 \%)$.

\footnotetext{
${ }^{4}$ Cabe destacar que, cuando se evalúa la capacidad cognitiva $Y$ de un adulto de acuerdo a las circunstancias $C$ de sus padres, se plantea una estimación: $Y=\alpha_{1} C+u$. Sin embargo, podría ser que en el proceso de formación de la capacidad cognitiva final, la variable $Y$ haya intervenido en la misma capacidad cognitiva en las etapas intermedias, de tal forma que en realidad el proceso verdadero es: $Y=\alpha_{2} C+\beta Y+v$. En este caso se plantea que $0<\beta<1 \cdot \beta$ es positivo porque la capacidad cognitiva inicial favorece la capacidad cognitiva final y $\beta<1$ porque de otra forma la participación de las circunstancias sería siempre la de reducir la capacidad cognitiva. Los coeficientes planteados guardan la relación $(1-\beta) Y=\alpha_{2} C+v=(1-\beta)\left(\alpha_{1} C+u\right)$, por lo que $\alpha_{1}=\frac{1}{1-\beta} \alpha_{2}$. Así, podría ser que las estimaciones de $\operatorname{GE}(0), \operatorname{GE}(1)$ y $\operatorname{GE}(2)$ estén sobreestimadas.
} 
Panorama ECONÓMICO, vol. VI, núm. 12, enero-junio de 2011

\section{TABLA 6}

\section{Características de los grupos con más desventajas por gasto en el consumo y nivel de habilidades cognitivas}

\begin{tabular}{|c|c|c|c|c|c|}
\hline \multirow{2}{*}{\multicolumn{2}{|c|}{$\begin{array}{l}\text { Variable dependiente } \\
\text { Ronda ENNVIH }\end{array}$}} & \multicolumn{2}{|c|}{$\begin{array}{l}\text { Gasto mensual en el } \\
\text { consumo del hogar }\end{array}$} & \multicolumn{2}{|c|}{$\begin{array}{c}\text { Nivel de habilidades } \\
\text { cognitivas }\end{array}$} \\
\hline & & 2002 & 2005 & 2002 & 2005 \\
\hline \multicolumn{2}{|c|}{ Circunstancias } & Porcentaje & Porcentaje & Porcentaje & Porcentaje \\
\hline \multirow{2}{*}{ Indígena } & Sí & 39.74 & 38.01 & 37.64 & 29.10 \\
\hline & No & 60.26 & 61.99 & 62.36 & 70.90 \\
\hline \multirow{2}{*}{$\begin{array}{l}\text { Lugar de } \\
\text { nacimiento }\end{array}$} & Ciudad, & 0.80 & 0.87 & 0.32 & 0.61 \\
\hline & $\begin{array}{l}\text { pueblo, ranchería, } \\
\text { ejido, hacienda o villa }\end{array}$ & 99.20 & 99.13 & 99.68 & 99.39 \\
\hline \multirow{3}{*}{$\begin{array}{l}\text { Ocupación } \\
\text { del padre }\end{array}$} & Agricultura & 82.81 & 86.86 & 87.20 & 64.98 \\
\hline & No-agricultura & 3.15 & 2.61 & 0.00 & 18.30 \\
\hline & No sabe & 14.04 & 10.53 & 12.80 & 16.73 \\
\hline \multirow{3}{*}{$\begin{array}{l}\text { Educación } \\
\text { del padre }\end{array}$} & Sin educación & 95.16 & 86.05 & 95.39 & 88.82 \\
\hline & Primaria & 4.84 & 13.62 & 4.35 & 10.49 \\
\hline & Más & 0.00 & 0.33 & 0.25 & 0.69 \\
\hline \multirow{3}{*}{$\begin{array}{c}\text { Educación } \\
\text { de la } \\
\text { madre }\end{array}$} & Sin educación & 97.28 & 96.73 & 99.71 & 97.98 \\
\hline & Primaria & 2.72 & 3.06 & 0.13 & 0.96 \\
\hline & Más & 0.00 & 0.16 & 0.16 & 1.06 \\
\hline
\end{tabular}

\section{CONCLUSIONES}

La teoría de igualdad de oportunidades de Roemer constituye una gran contribución a la literatura y discusión de la justicia distributiva. A partir de esta teoría se han desarrollado numerosos estudios acerca del concepto de igualdad de oportunidades. Ferreira y Gignoux (2008) plantean una medida para calcular la desigualdad de oportunidades en los salarios, el ingreso y el consumo de los hogares en seis países de Latinoamérica. El presente trabajo utiliza la medida propuesta por ese artículo, un índice que mide la proporción de desigualdad atribuida a circunstancias predeterminadas, como el lugar de nacimiento del individuo y sus antecedentes familiares. 
Alférez EsPerón / MAYer-Foulkes Desigualdad de oportunidades en México:

Impacto sobre el consumo y sobre las habilidades cognitivas

Tomando las dos rondas de la Encuesta Nacional de Niveles de Vida de los Hogares, se construyeron índices asociados a la desigualdad de oportunidades en México. De la ronda 2002 y 2005 se obtuvieron datos, a nivel individuo, del lugar de nacimiento, el nivel de escolaridad de la madre y del padre, la ocupación del padre e información relativa a la pertenencia a algún grupo indígena. Con esta información se llevó a cabo una partición de la población en grupos de acuerdo a las circunstancias que los caracterizan y se estimó la desigualdad entre cada grupo. En la primera estimación se utilizó como variable dependiente el gasto mensual en el consumo del hogar y en la segunda estimación se utilizó el nivel de habilidades cognitivas.

Los resultados de la estimación de cotas inferiores de la desigualdad en el consumo del hogar mostraron que, en 2002 la desigualdad de oportunidades era aproximadamente $35.9 \%$ respecto a la desigualdad total y en 2005 de $34.7 \%$. Al considerar cada variable del conjunto de circunstancias por separado se encontró que, los grados de escolaridad de los padres junto con el lugar de nacimiento determinan gran parte de la desigualdad de oportunidades de los individuos. Los resultados de la estimación para la desigualdad en el nivel de habilidades cognitivas mostraron que, en 2002 la desigualdad de oportunidades era aproximadamente $55.2 \%$ respecto a la desigualdad total y en 2005 de $37 \%$. Al considerar cada variable del conjunto de circunstancias por separado, se encontró, de nuevo, que el lugar de nacimiento y la escolaridad de los padres determinan la mayor parte de la desigualdad de oportunidades de los individuos.

A partir de los subgrupos obtenidos, se construyó un perfil de oportunidades que muestra la estructura social de los grupos con más desventajas en el consumo y en el nivel de habilidades cognitivas. Los resultados muestran que, los individuos con más desventajas económicas son aquellos que están compuestos por individuos que no nacieron en una ciudad, cuyos padres no reportan ningún nivel de escolaridad y cuyo padre trabajaba en una actividad relacionada con la agricultura.

Las dos estimaciones que se llevaron a cabo muestran que existe una gran proporción de la desigualdad total que está determinada por la desigualdad de oportunidades. Esto tiene un impacto significativo en el consumo de los hogares y, en 
mayor medida, en las habilidades cognitivas de los individuos cuando llegan a la edad adulta. El perfil de oportunidades que aquí se muestra puede ser útil para enfocar futuras políticas económicas dirigidas a reducir la desigualdad que proviene de las circunstancias que están fuera del control y del esfuerzo de los individuos, de tal manera que las oportunidades en México sean más inclusivas en el proceso de desarrollo humano y crecimiento económico.

\section{BIBLIOGRAFÍA}

Arrow, Kenneth, Samuel Bowles y Steven N. Durlauf (2000), Meritocracy and economic inequality. Princeton University Press.

Alkire, Sabina (2010), "Human Development: Definitions, Critiques, and Related Concepts", Human Development Research Paper HDRP/2010/01, New York: United Nations Development Programme, Human Development Report Office.

Barros, Ricardo Paes de, Francisco H. G. Ferreira, José Molinas Vega y Jaime Saavedra Chanduvi (2009), "Measuring Inequality of Opportunities in Latin America and the Caribbean", Washington, DC: Palgrave Macmillan and the World Bank.

Behrman, Jere R., Susan W. Parker y Petra E. Todd (2007), "Do School Subsidy Programs Generate Lasting Benefits? A Five-Year Follow-Up of Oportunidades Participants", University of Pennsylvania, mimeo.

Bossert, Walter (1995), "Redistribution Mechanisms Based on Individual Characteristics", Mathematical Social Sciences, 29: 1-17.

Bourguignon, François, Francisco H.G. Ferreira y Marta Menéndez (2007), "Inequality of Opportunity in Brazil”, Review of Income Wealth, 53 (4): 585-618.

Bourguignon, François, Francisco H.G. Ferreira y Michael Walton (2007), "Equity, Efficiency and Inequality Traps: A research agenda", Journal of Economic Inequality 5: 235-256.

Checchi, Daniele y Vitoroco Peragine (2005), "Regional Disparities and Inequality of Opportunity: the Case of Italy", IZA Discussion Paper No. 1874/2005. 
Alférez EsPerón / MAYer-Foulkes Desigualdad de oportunidades en México: Impacto sobre el consumo y sobre las habilidades cognitivas

Cogneau, Denis y Jérémie Gignoux (2007), "Earnings Inequalities and Educational Mobility in Brazil over two Decades" en Klasen S. y Nowak-Lehmann (eds.), Poverty, Inequality and Policy in Latin America, CESifo Seminar Series, Massachusetts Institute of Technology (MIT) Press.

Dworkin, Ronald (1981), What is equality? Part 1: Equality of welfare. Part 2: Equality of resources. Philosophy \& Public Affairs 10, 185-246 y 283-345.

Ferreira, Francisco H.G. y Jérémie Gignoux (2008), "The Measurement of Inequality of Opportunity: Theory and an application to Latin America", World Bank Policy Research Working Paper, \#4659.

Fleurbaey, Marc (1995), “The requisites of equal opportunity”. En W.A. Barnett, H. Moulin, M. Salles, N.J. Schofield (Eds.), Social Choice, Welfare and Ethics, pp. 37-53, Cambridge University Press.

Gignoux, Jérémie (2009), "Educational Expansion and Inequality of Earnings Opportunities: Recent Trends in Latin American Countries", Research for Public Policy, Human Development, HD-10-2009, RBLAC-UNDP, New York.

Lefranc, Arnaud, Nicolas Pistolesi y Alain Trannoy (2006), "Inequality of opportunities vs. inequality of outcomes: Are Western societies all alike?" ECINEQ Society for the Study of Economic Inequality working Paper, \#54.

Lefranc, Arnaud, Nicolas Pistolesi y Alain Trannoy (2009), "Equality of Opportunity and Luck: Definitions and testable conditions, with an application to income in France", Journal of Public Economics: 1189-1207.

Lucas, J. R. (1995), Responsibility. Oxford: Clarendon Press.

Mayer, David y Gabriela Cordourier (2001), "La Brecha Salarial y la Teoría de Igualdad de Oportunidades. Un Estudio de Género para el Caso Mexicano", El Trimestre Económico, vol. LXVIII(1), num. 269, pp. 71-107.

Mayer, David, María Fernanda López Olivo y Edson Serván Mori (2008), “Habilidades cognitivas: transmisión intergeneracional por niveles socioeconómicos", Estudios Económicos, El Colegio de México, Centro de Estudios Económicos, vol. 23(1), pp. 129-156.

Roemer, John E. (1993), "A Pragmatic Theory of Responsibility for the Egalitarian Planner", Philosophy and Public Affairs, 22: 146-166. 
Panorama ECONÓMICO, vol. VI, núm. 12, enero-junio de 2011

Roemer, John E. (1998), Equality of Opportunity. Cambridge, MA: Harvard University Press.

Roemer, John et al. (2003), "To What Extent do Fiscal Systems Equalize Opportunities for Income Acquisition among Citizens?", Journal of Public Economics, 87.

Roemer, John E. (2006), "Review Essay, 'The 2006 world development report: Equity and Development", Journal of Economic Inequality 4: 233-244.

Rubalcava, Luis y Graciela Teruel (2004), "The role of maternal cognitive ability on child health", Economics and Human Biology, Elsevier, vol. 2(3), pp. 439-455.

Rubalcava, Luis y Graciela Teruel (2006), “User's Guide for the Mexican Family Life Survey: The First Wave", disponible en <http://www.mxfls.cide.edu>.

Van de Gaer, Dirk (1993), "Equality of opportunity and investment in human capital", Catholic University of Leuven, Faculty of Economics, no. 92.

Van de Gaer, Dirk, Erik Schokkaert y Michel Martinez (2001), "Three Meanings of Intergenerational Mobility", Economica, 68: 519-537.

World Bank (2005), World Development Report 2006: Equity and Development, Washington, DC: The World Bank and Oxford University Press. 
Alférez Esperón / MaYer-Foulkes

Desigualdad de oportunidades en México:

Impacto sobre el consumo y sobre las habilidades cognitivas

\section{ANEXOS}

\section{TABLA 7}

\section{Estadística descriptiva de la muestra}

\begin{tabular}{|c|c|c|}
\hline \multirow{2}{*}{ Variables (circunstancias) } & \multicolumn{2}{|c|}{ Porcentajes } \\
\hline & 2002 & 2005 \\
\hline \multicolumn{3}{|l|}{ Lugar de nacimiento } \\
\hline Ciudad & 28.14 & 26.07 \\
\hline $\begin{array}{l}\text { Áreas rurales } \\
\text { (pueblo, }\end{array}$ & & \\
\hline $\begin{array}{l}\text { ranchería, ejido, } \\
\text { hacienda, villa, } \\
\text { otro) }\end{array}$ & 71.86 & 73.07 \\
\hline \multicolumn{3}{|l|}{ Género } \\
\hline Hombre & 48.45 & 49.87 \\
\hline Mujer & 51.55 & 50.13 \\
\hline \multicolumn{3}{|l|}{ Pertenencia a una etnia } \\
\hline Sí & 12.89 & 13.15 \\
\hline No & 87.11 & 86.85 \\
\hline \multicolumn{3}{|l|}{ Educación de la madre } \\
\hline Sin educación & 64.5 & 67.21 \\
\hline Primaria completa & 30.94 & 28.97 \\
\hline Secundaria o más & 4.57 & 3.82 \\
\hline \multicolumn{3}{|l|}{ Educación del padre } \\
\hline Sin educación & 61.53 & 64.33 \\
\hline Primaria completa & 32.31 & 30.41 \\
\hline Secundaria o más & 6.16 & 5.27 \\
\hline \multicolumn{3}{|l|}{ Ocupación del padre } \\
\hline $\begin{array}{l}\text { Obrero o } \\
\text { empleado en el } \\
\text { sector } \\
\text { agropecuario }\end{array}$ & 31.13 & 33.49 \\
\hline $\begin{array}{l}\text { Empleado en otro } \\
\text { sector }\end{array}$ & 24.21 & 20.73 \\
\hline No se sabe & 44.66 & 45.77 \\
\hline \multicolumn{3}{|l|}{ Variables de ventaja } \\
\hline \multicolumn{3}{|l|}{ Gasto mensual a nivel hogar } \\
\hline Media & 3894.795 & 4058.636 \\
\hline e.s. & {$[4352.37]$} & [4399.25] \\
\hline \multicolumn{3}{|l|}{ Resultados de prueba Raven } \\
\hline Media & 0.4601929 & 0.52562 \\
\hline e.s. & [0.249] & {$[0.240]$} \\
\hline
\end{tabular}




\section{Figura 5}

\section{Desigualdad de oportunidades por circunstancias} en el consumo del hogar.

Comparación entre rondas 2002 y 2005 por estimadores
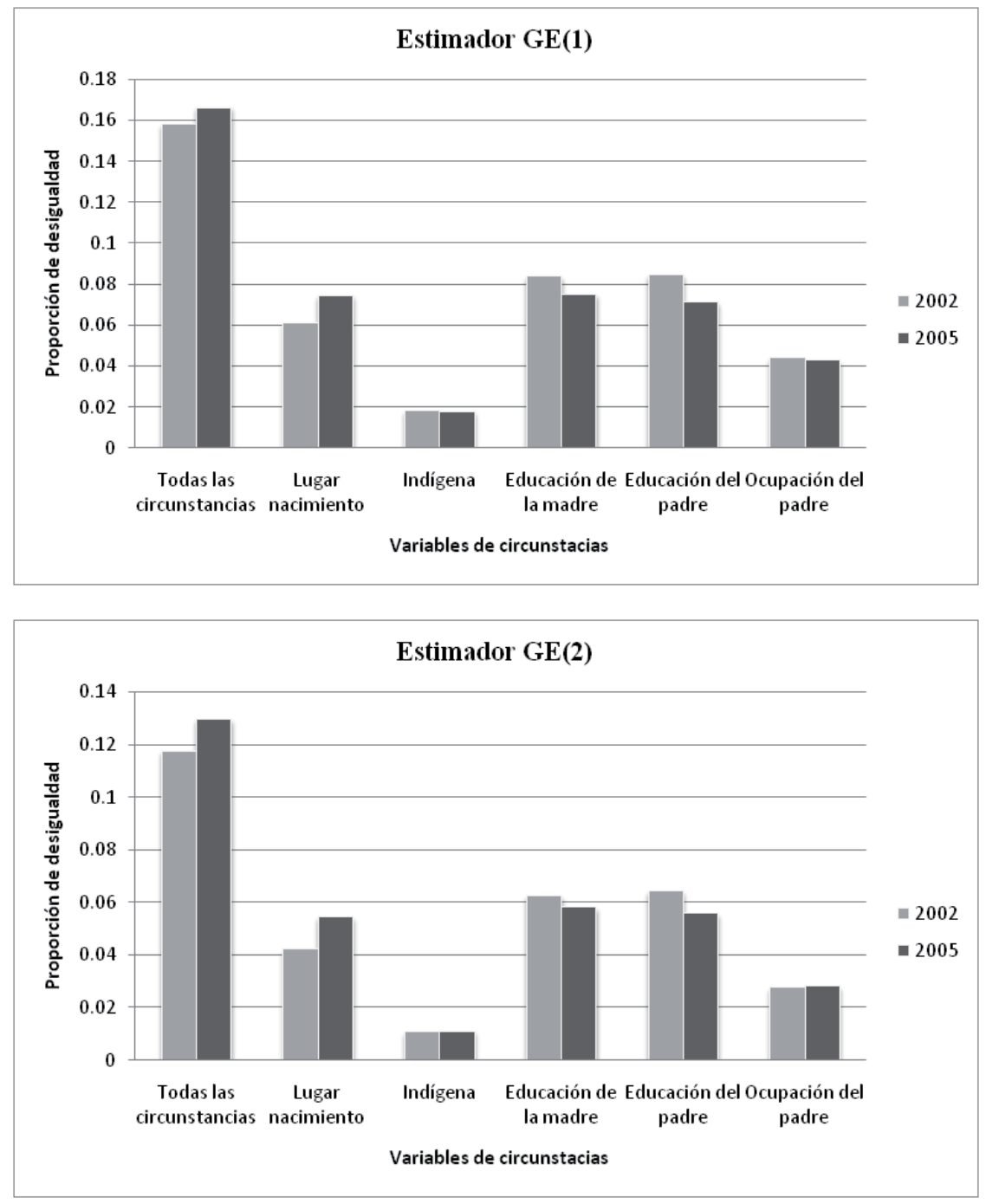
Alférez EsPerón / MAYer-Foulkes Desigualdad de oportunidades en México: Impacto sobre el consumo y sobre las habilidades cognitivas

Figura 6

Desigualdad de oportunidades por circunstancias en el nivel de habilidades cognitivas.

Comparación entre rondas 2002 y 2005 por estimadores
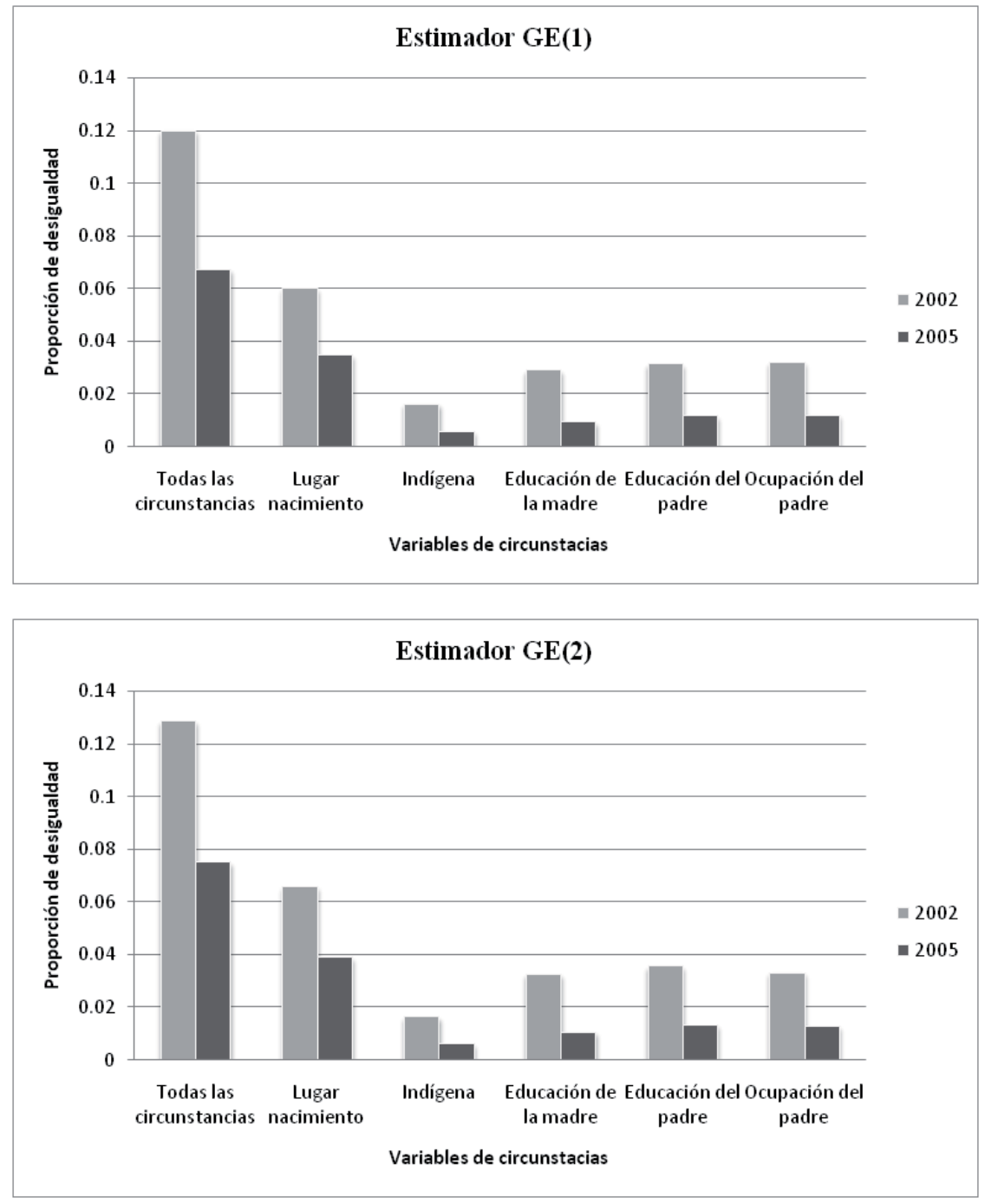